\title{
Deformation potentials of CdSe quantum dots
}

\author{
Jingbo Li and Lin-Wang Wang* \\ Computational Research Division, Lawrence \\ Berkeley National Laboratory, Berkeley, CA 94720
}

(Dated: June 1, 2004)

\begin{abstract}
The size dependent deformation potentials of CdSe quantum dots are studied by first principle and semi-empirical pseudopotentials calculations. We find that the amplitude of the quantum dot deformation potential is only slightly larger than the bulk value, and this increase is mostly caused by the off $\Gamma$ point deformation potentials in the bulk, which are larger in amplitude than the $\Gamma$ point deformation potential.
\end{abstract}

PACS numbers: 71.15.Dx, 73.22.-f, 81.40.Vw

*Electronic address: lwwang@lbl.gov 
One way to study the electronic structure of semiconductor quantum dots (QDs) is to apply hydrostatic pressure. Under pressure, solid-solid phase transitions have been observed in CdSe QD, [1-5] and direct-to-indirect band gap transitions have been reported for $\mathrm{InP}$ and Si QDs [6-8]. Another approach is to measure the size dependence of the QD band gap pressure coefficients. [9-13] Unfortunately, the available experimental data for QD band gap pressure coefficients are often controversial. For example, for CdSe QDs, Alivisatos et.al [9] have reported a band gap pressure coefficient of $45 \mathrm{meV} / \mathrm{GPa}$, which is in the range of experimental bulk pressure coefficients $\sim 43-56 \mathrm{meV} / \mathrm{GPa}[13]$. But Li et al. [10, 11] and Shan et al. [13] have reported much smaller pressure coefficients around $27 \mathrm{meV} / \mathrm{GPa}$, meanwhile Meulenberg and Strouse [12] have reported a surprisingly large pressure coefficient around $82 \mathrm{meV} / \mathrm{GPa}$. Along with these experimental uncertainties, there is a lack of understanding for how and why the band gap pressure coefficient of the QD will change from its bulk value. In this paper, we will use $a b$ initio calculations to address this question. We will study the size-dependent deformation potentials for CdSe QDs.

We will use the local density approximation (LDA) of the density functional theory (DFT) to calculate the electronic structure of thousand atom CdSe QDs. Although the LDA has a band gap error, but its deformation potential does reproduce the experimental results well. We will also use the semiempirical pseudopotential method (SEPM) to compare with our LDA results. To calculate the thousand atom QDs under LDA, we will use the newlydeveloped $a b$ initio charge patching method. Using this method, the QD charge density $\rho(r)$ will be generated from motifs without doing self-consistent calculations. The potential $V(r)$ of QDs will then be calculated from $\rho(r)$ using the LDA formula. Next, the single-particale Schrödinger's equation $H \psi_{i}=\epsilon_{i} \psi_{i}$ will be solved using the folded spectrum method [14] for a few states near the band gap. The details of this procedure are reported in Ref. [15], and the typical accuracy for the eigen energies is around 20-30 meV compared to direct LDA results.

We have first checked the self-consistent plane-wave (PW) pseudopotential calculations for bulk CdSe. We have tested two pseudopotentials, one without the $4 \mathrm{~d}$ states in the valence electron (PW) using 35 Ryd planewave cutoff, one with the 4d states (PW+d) using 60 Ryd planewave cutoff. The deformation potentials $\alpha_{v}=\frac{d E_{g}}{d \ln v}$ (where $E_{g}$ is the band gap, $V$ is the volume) for the bulk from these two pseudopotentials are listed in Table I, along with the all electron linear augmented planewave (LAPW) results [18], fitted SEPM results, and the 
experimental values $[18,19]$. Also reported in Table I are the experimental and calculated bulk moduli. We see that, the PW+d results agree very well with the LAPW results. But, ironically, the PW results without the $4 \mathrm{~d}$ states agree better with the experiments. The removal of the $4 \mathrm{~d}$ states has accidentally corrected the LDA error. We have thus chosen the PW method in the following calculation.

The size-dependent hydrostatic deformation potentials (in eV) of CdSe QDs are calculated by direct $a b$ initio self-consistent calculation (for the three smallest QDs, upto 200 atoms), ab initio charge patching method, and SEPM method. The results are shown in Fig. 1. The crystal structures for all the CdSe QDs are zinc-blende. As one can see from Fig.1, for the three smallest QDs $\left(\mathrm{Cd}_{43} \mathrm{Se}_{44}, \mathrm{Cd}_{79} \mathrm{Se}_{68}\right.$, and $\left.\mathrm{Cd}_{107} \mathrm{Se}_{104}\right)$ where direct selfconsistent calculations are performed, the charge patching results agree well with the direct self-consistent results. This means that the charge patching method can be used to study the pressure dependence of the system. It is also shown in Fig.1 that the SEPM results agree well with the LDA results.

Fig. 1 illustrates that the amplitude of the deformation potential $\alpha_{v}$ of the QDs increases as the size of dots decrease. At the smallest quantum dot of $1.7 \mathrm{~nm}$ in diameter, $-\alpha_{v}$ has increased $20 \%$ from its bulk value. However, around $4 \mathrm{~nm}$ where most experimental measurements are carried out, this increase is only about $3 \%$. The measured band gap pressure coefficient is a result of the product between the deformation potential and the Young's moduli. If one assumes that the Young's moduli for a QD is the same as in the bulk, then from our calculation, the band gap pressure coefficient should also be close to the bulk values. This conclusion is similar to the experimental results by Alivisatos, et.al [9], but not consistent with the other experimental results [10-13]. The possible change of the QD Young's moduli from its bulk value has to be investigated seperately using total energies, and different surface passivations might also play a role. Here, we have used a pseudo-hydrogen atom passivation to remove all the surface dangling bonds. [15] This passivation is good to describe the internal electronic structure of the QD, but its effects on the total energy and surface tension need to be studied more carefully. Thus, we will defer the study of the QD Young's moduli to a different work.

One interesting question is: what cause the slight increase of the QD deformation potential amplitude? To answer this question, we have analysized the QD wavefunctions and eigen energies. In the reciprocal space, a QD eigenfunction $\psi_{i}$ can be projected into Bloch 
functions $\left\{u_{n k} e^{i k r}\right\}$ of band index $n$ and the supercell allowed reciprocal vector $\mathbf{k}$ within the first Brillouin zone. After summing up band index $n$, one gets a projection function, $W_{i}(\mathbf{k})=\sum_{\mathbf{n}=\mathbf{1}}^{\infty}\left|\left\langle\psi_{\mathbf{i}}(\mathbf{r}) \mid \mathbf{u}_{\mathbf{n k}}(\mathbf{r}) \mathbf{e}^{\mathbf{i} \mathbf{k r}}\right\rangle\right|^{\mathbf{2}}$, where $W_{i}(\mathbf{k})$ can be viewed as an $k$-spectral weight. Fig. 2(a) shows the spectral weight $W_{c}(\mathbf{k})$ for the SEPM conduction band minimum (CBM) state of the $\mathrm{D}=3.75 \mathrm{~nm}$ QD. Notice that, in this case, the $\Gamma$ point accounts only for $21.9 \%$ of the total weight. Thus, most of the weight comes from other k-points. For the CBM states, it turns out most of the spectral contributions come from the bulk first conduction band. As a result, the eigen energy for the QD CBM state can be approximated as:

$$
E_{C B M}=\sum_{k} W_{c}(\mathbf{k}) E(\mathbf{k})
$$

where $E(\mathbf{k})$ is the bulk first conduction band energy at $\mathbf{k}$ point. This formula is tested using the SEPM calculation. The SEPM instead of the LDA calculations are used in the analysis since the SEPM does not have the technical complications of CBM alignment for different QDs. The results from the direct calculations and Eq. (1) are listed in Table.II as the first and second columns respectively. As one can see, except for the very small QDs where the surface effects might be important, Eq. (1) captures the essense of the quantum confinement effect.

Using Eq. (1), the QD deformation potential can then be calculated as:

$$
\frac{d E_{C B M}}{d \ln V}=\sum_{k}\left[\frac{d W_{c}(\mathbf{k})}{d \ln V} E(\mathbf{k})+W_{c}(\mathbf{k}) \frac{d E(\mathbf{k})}{d \ln V}\right],
$$

where $\mathrm{V}$ is the volume of the QD. The derivations in Eq. (2) regarding to $\ln V: W_{c}^{\prime} \equiv \frac{d W_{c}(\mathbf{k})}{d \ln V}$ and $E^{\prime}(k) \equiv \frac{d E(\mathbf{k})}{d \ln V}$ are done under an fixed relative reciprocal vector $\mathbf{k} /(\mathbf{2} \pi / \mathbf{a})$ ( $a$ is the lattice constant), instead of a fixed absolute reciprocal vector $|\mathbf{k}|$.

To use Eq. (2), the bulk deformation potentials $E^{\prime}(k)$ for off $\Gamma k$-points have to be calculated first. This quantity is shown in Fig.3 for the first conduction band. Using such results as in Fig.3, we have calculated the two terms in Eq. (2), and listed the results in Table.II. The sum of these two terms according to Eq. (2) is shown in Fig.4, which is very close to the directly calculated SEPM QD results.

From Table II, we see that, for the QDs, the biggest change from the bulk value is from the $\sum W_{c} E^{\prime}$ term. The amplitude of this term increases since the $-\alpha_{v}$ from off $\Gamma$ point is larger then the $\Gamma$ point value, as shown in Fig.3. Towards the $\mathrm{X}$ point, the $-\alpha_{v}$ drops significantly, eventually into negative regime. However, in the direct band gap CdSe QDs, most weights 
$W_{c}(k)$ are within a sphere of $|\mathbf{k}|<\frac{2 \pi}{a}(0.25,0.25,0.25)$ as shown in Fig.2(a). Thus, the near $\mathrm{X}$ point drop of $-\alpha_{v}$ have no effect. This explains why the CdSe QD deformation potential amplitude is always larger than the bulk value.

The amplitude of the first term $\sum W_{c}^{\prime} E$ in Eq. (2) is about a quarter of the change of the second term $\sum W_{c} E^{\prime}$ from its bulk value, but with an opposite sign. This term comes from the change of the spectrum weight when the QD is under pressure. This change is illustrated in Fig.2(b) (for the k points with increased $W_{c}(k)$ ) and Fig.2(c) (for the k points with decreased $\left.W_{c}(k)\right)$. We see that, under the pressure, the $\Gamma$ point weight increases, while the weights for most k-points surrounding the $\Gamma$ point decreases. But for very large k-points, their weights also increase slightly. This complicated pattern leads the results of $\sum W_{c}^{\prime} E$ in Table.II. Notic that, in an effective mass theory, if the barrier wall is infinity, then the weight $W_{c}(k)$ should not change. For a finite fixed barrier wall, under the pressure, the increased band gap will lead to an increased effective mass. As a result, the wavefunction will have a stronger confinement. Then the $W_{c}(\Gamma)$ should decrease instead of increase. Thus, the pattern of $W_{c}(k)$ change shown in Fig.2 cannot be described by simple effective mass theory.

This work was supported by U.S. Department of Energy under Contract No. DE-AC0376SF00098. This research used the resources of the National Energy Research Scientific Computing Center.

[1] K. Jacobs, D. Zaziski, E. C. Scher, A. B. Herhold, A. P. Alivisatos, Science 293, 1803 (2001).

[2] J. N. Wickham, A. B. Herhold, A. P. Alivisatos, Phys. Rev. Lett. 84, 923 (2000).

[3] S. H. Tolbert, A. B. Herhold, L. E. Brus, A. P. Alivisatos, Phys. Rev. Lett. 76, 4384 (1996).

[4] S. H. Tolbert, A. P. Alivisatos, J. Chem. Phys. 102, 4642 (1995).

[5] C. Molteni, R. Martonak, M. Parrinello, J. Chem. Phys. 114, 5358 (2001).

[6] S. H. Tolbert, A. B. Herhold, C. S. Johnson, A. P. Alivisatos, Phys. Rev. Lett. 73, 3266 (1994).

[7] C. J. Lee, A. Mizel, U. Banin, M. L. Cohen, A. P. Alivisatos, J. Chem. Phys. 113, 2016 (2000).

[8] H. Fu and A. Zunger, Phys. Rev. Lett. 80, 5397 (1998).

[9] A. P. Alivisatos, T. D. Harris, L. E. Brus, A. Jayaraman, J. Chem. Phys. 89, 5979 (1988).

[10] J. Li, G. H. Li, J. B. Xia, J. B. Zhang, Y., Lin, and X. R. Xiao, J. Phys.: Condens. Matter 
13, 2033 (2001).

[11] B. S. Kim, M. A. Islam, L. E. Brus, I. P. Herman, J. Appl. Phys. 89, 8127 (2001).

[12] R. W. Meulenberg and G. F. Strouse, Phys. Rev. B 66, 035317 (2002).

[13] W. Shan et al., Appl. Phys. Lett. 84, 67 (2004); W. Shan (private communication).

[14] L. W. Wang and A. Zunger, J. Chem. Phys. 100, 2394 (1994).

[15] L. W. Wang and J. Li, Phys. Rev. B 69, 153302 (2004); J. Li and L. W. Wang, Appl. Phys. Lett. 84, 3648 (2004).

[16] J. Li and L. W. Wang, Nano Lett. 3, 101 (2003); Nano Lett. 3, 1357 (2003); Nano Lett. 4, 29 (2003).

[17] L. W. Wang and A. Zunger, Phys. Rev. B51, 17398 (1995), and references therein.

[18] S. H. Wei and A. Zunger, Phys. Rev. B60, 5404 (1999), and references therein.

[19] A. Blacha, H. Presting, and M. Cardona, phys. stat. sol. (b)126, 11 (1984). 
TABLE I: Calculated and experimental bulk moduli $B_{0}$ (in GPa), deformation potential $\alpha_{v}$ (in eV) of zinc-blende CdSe.

\begin{tabular}{cccccc}
\hline \hline & SEPM & $\mathrm{PW}^{a}$ & $\mathrm{PW}^{a} \mathrm{~d}^{a}$ & LAPW $^{b}$ & Expt. $^{b c}$ \\
\hline$B_{0}$ & 56.5 & 58.7 & 59.2 & 53.0 \\
$\alpha_{v}$ & -2.98 & -2.98 & -2.05 & -1.96 & -3.0 \\
\hline \hline
\end{tabular}

${ }^{a}$ present calculations

${ }^{b}$ Ref. [18]

${ }^{c}$ Ref. [19]

TABLE II: Analysis of QDs' deformation potential by Eqs.(1) and (2).

\begin{tabular}{ccccc}
\hline \hline diameter $(\mathrm{nm})$ & $\mathrm{E}_{C B M}$ & $\sum W_{c} E$ & $\sum W_{c}^{\prime} E$ & $\sum W_{c} E^{\prime}$ \\
\hline 1.67 & -2.056 & -1.537 & 0.319 & -4.245 \\
1.99 & -2.201 & -2.099 & 0.200 & -3.870 \\
2.25 & -2.303 & -2.228 & 0.180 & -3.710 \\
2.51 & -2.361 & -2.301 & 0.130 & -3.590 \\
3.24 & -2.499 & -2.459 & 0.070 & -3.315 \\
3.75 & -2.569 & -2.536 & 0.036 & -3.190 \\
4.15 & -2.581 & -2.567 & 0.029 & -3.130 \\
$\infty$ & -2.827 & -2.827 & 0.0 & -3.050 \\
\hline \hline
\end{tabular}




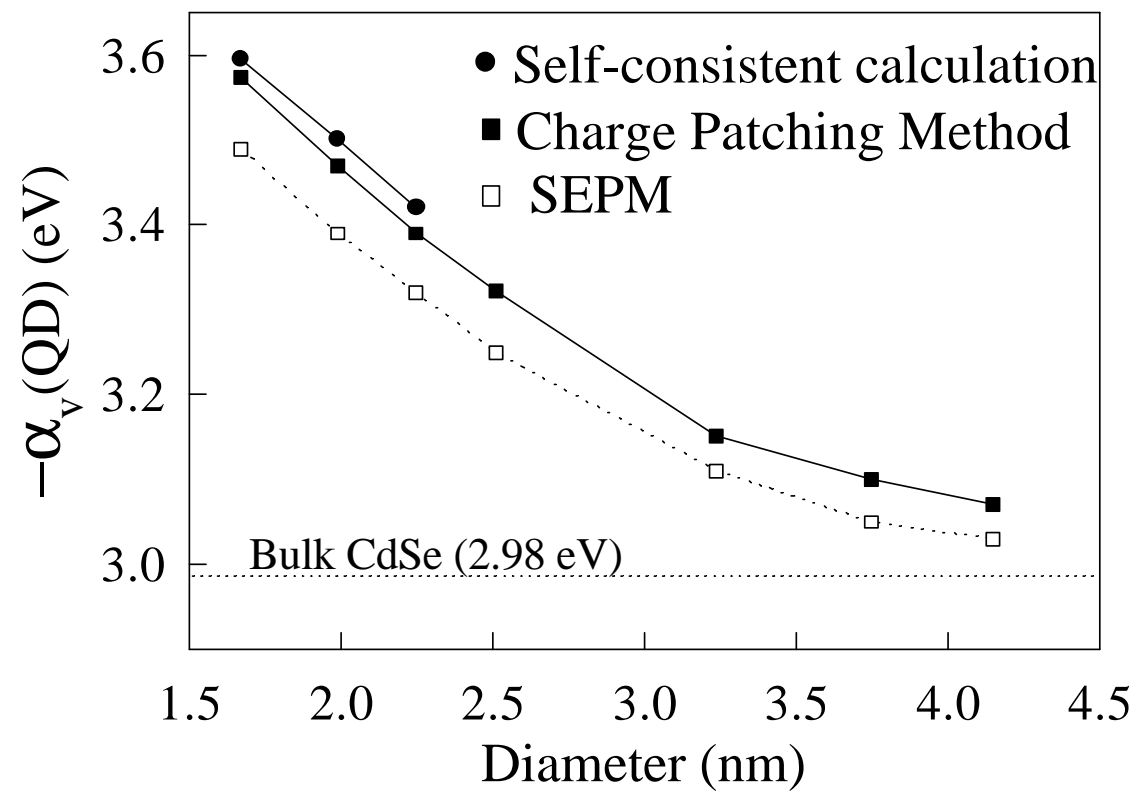

FIG. 1: The size dependence of $-\alpha_{v}$ of CdSe QDs calculated by self-consistent calculation (fulldots), charge patching method (full-squares), and SEPM (open-squares). 


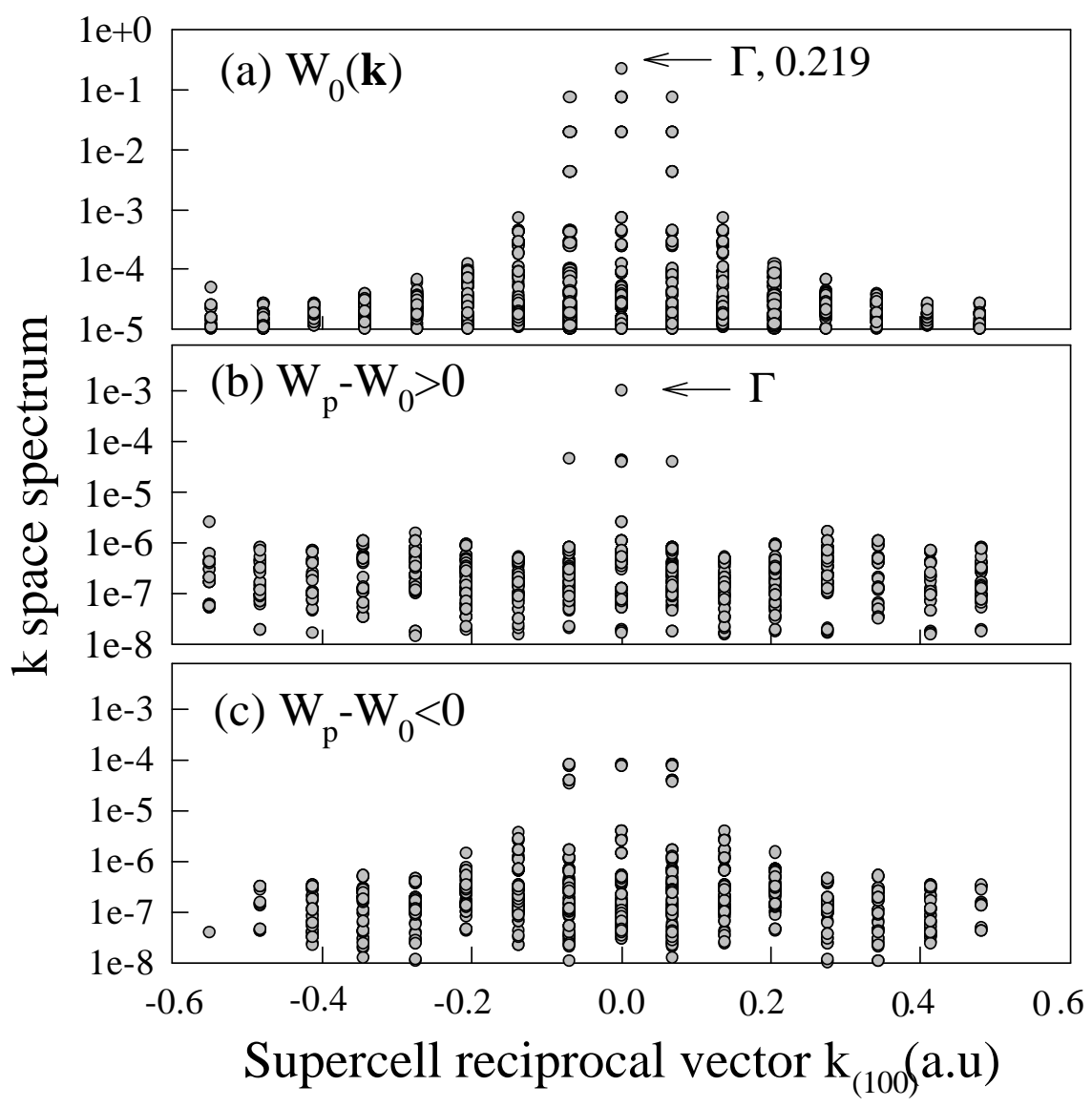

FIG. 2: $k$ space spectrum of the CBM state of $3.75 \mathrm{~nm}$ diameter QDs. $W_{0}$ denotes the weight under zero pressure, while $W_{p}$ denotes the weight under pressure. 


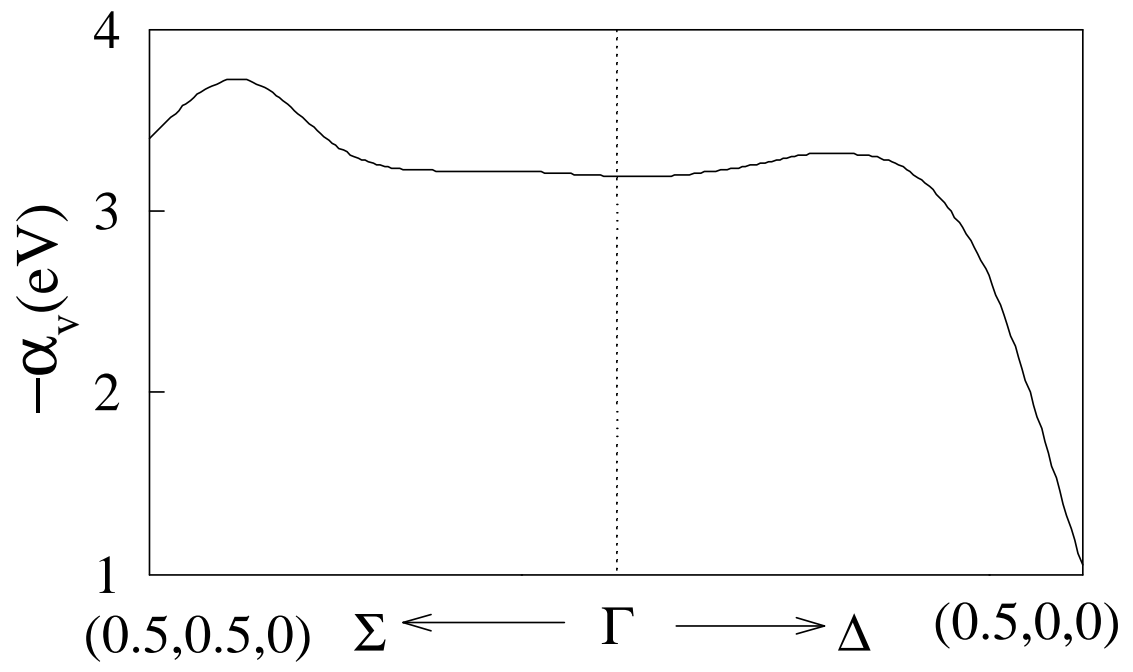

FIG. 3: $-\alpha_{v}$ of CBM state of bulk CdSe in the $\mathrm{k}$ space. The wave vector is in unit of $\frac{2 \pi}{a}$.

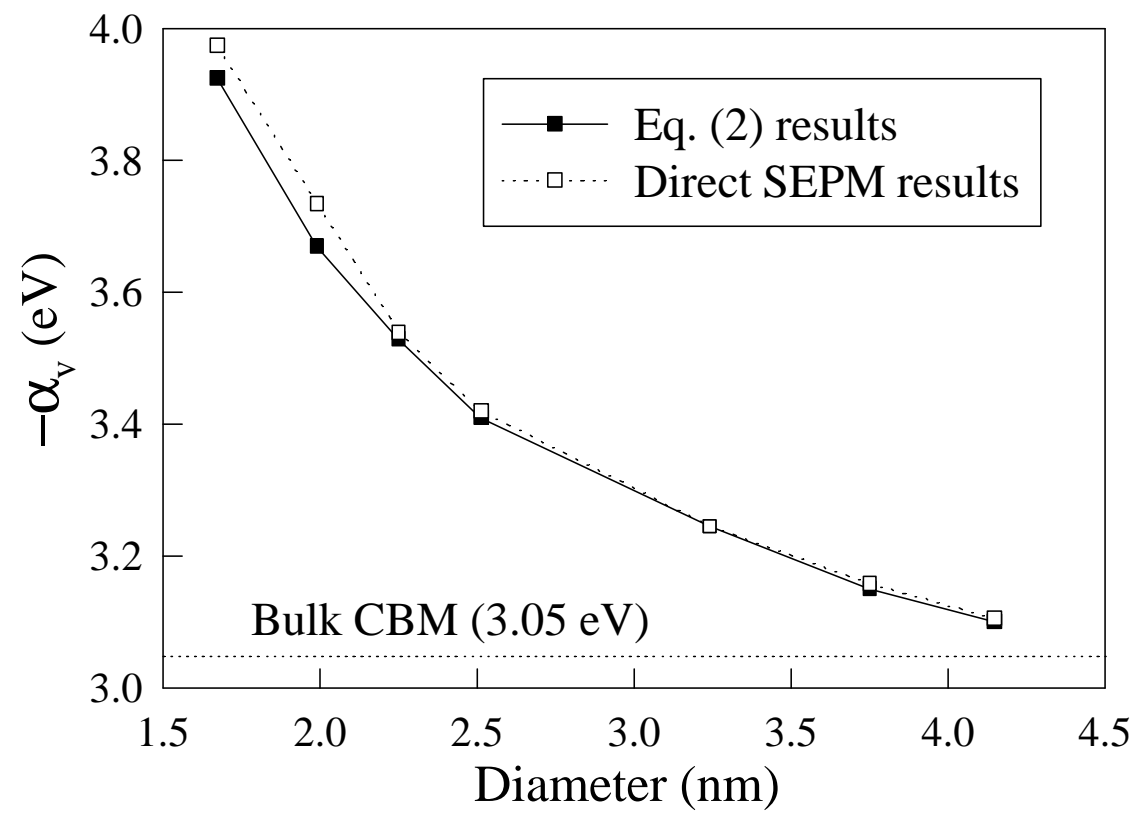

FIG. 4: The size dependence of $-\alpha_{v}$ of CBM state of CdSe QDs. 